\title{
Acceso, uso y actitud de la tecnología en las escuelas de Educación Primaria en la Región de Murcia
}

\author{
Raúl Céspedes Ventura $\left(^{*}\right)$ y Javier Ballesta Pagán (*) \\ $\left.{ }^{*}\right)$ Universidad de Murcia - España
}

\section{RESUMEN}

La Sociedad de la Información demanda competencias específicas a sus actuales y futuros ciudadanos, entre ellas, la competencia digital. La tecnología debe estar presente en las aulas, pero la toma de decisiones, respecto a cómo hacer que esa presencia sea efectiva, no es tarea fácil, necesitando estudios que identifiquen indicadores y evalúen el estado de dicha integración, señalando lo que funciona bien y lo que se debe mejorar. Este estudio consta de dos etapas, una encuesta y un grupo de discusión. La Encuesta Escolar: Educación y TIC se realizó en 2015 desde la Universidad de Murcia con el objetivo de definir la situación de la integración de la tecnología en los centros escolares de la etapa de Educación Primaria. La encuesta se aplicó a todos los centros de la Región de Murcia encuestando tanto a directores como a tutores y realizándose íntegramente online. En este artículo se presentan los principales resultados y conclusiones de la encuesta, así como recomendaciones para administradores educativos de cualquier nivel.

Palabras Clave: evaluación de la educación, tecnología, tecnología de la información, política educacional, estrategias educativas.

\section{Access, use and attitudes to technology in Primary Education schools in the Region of Murcia}

\section{ABSTRACT}

Society of Information demands its current and future citizens specific competences, including digital competence. Technology must be present in the classrooms but making decisions about how to make that presence effective is not an easy task, needing studies that identify indicators and evaluate the status of such integration, pointing out what really works and what that must be improved. This study consists of two stages, a survey and a discussion group. The School Survey: Education and ICT in the Region of Murcia was conducted in 2015 at the University of Murcia with the aim of defining the situation of the integration of technology in schools of the Primary Education. The survey was applied to all the schools of the Region of Murcia surveying both directors and tutors and conducted entirely online. This article presents the main findings and conclusions of the survey, as well as some recommendations for educational administrators of any level.

Keywords: educational evaluation, technology, information technology, educational policy, educational strategies.

\section{Introducción}

La Sociedad de la Información nos ofrece un acceso masivo a datos, a información, pero sin garantías de lograr conocimiento. El exceso de información, mensajes sin contenido, falta de actitud crítica y un sinfín de factores limitan que esa información llegue en algún momento a hacer competentes a las personas (Sancho y Hernández, 2018). Por otro lado, los ordenadores, portátiles, tablets y smartphones son cada vez más importantes en educación como herramientas que añaden portabilidad y acceso tanto a alumnos como a docentes (Prensky, 2004; Pérez Tornero y Pi, 2014; Norris, Hossain y Soloway, 2011; De Pablos, 2010; Camacho y Esteve, 2018; Haßler, Major, y Hennessy, 2016; Biagi y

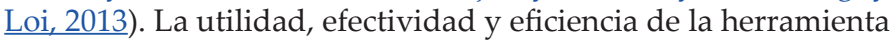

es determinada por unas condiciones técnicas y organizativas adecuadas (Area, 2010; Savill-Smith, 2005; Condie y Munro, 2007; Rodríguez, Nussbaum, y Dombrovskaia, 2013). Por tanto, la integración de tecnología debe seguir un plan establecido y contar con una organización detallada de todos los elementos implicados en el proceso para contribuir así al desarrollo de las competencias clave establecidas a nivel internacional, y dentro de estas competencias, especialmente la competencia digital, en el uso crítico y aprovechamiento de los medios digitales para el aprendizaje, el trabajo, el ocio y la comunicación (Valle y Manso, 2013).

En los últimos años son numerosos los estudios que se han centrado en la integración de las TIC en los centros (Colás, De Pablos y Ballesta, 2018). Uno de los más recientes es The Survey 
of Schools: ICT in Education. Benchmarking Access, Use and Attitudes to Technology in Europe's Schools (ESSIE 2011), estudio que destaca los problemas de infraestructura como uno de los obstáculos más importantes en la integración de las TIC en el aula, sobre todo en cuanto a insuficiencia o inoperatividad del equipamiento. También apunta al hecho de que las actividades basadas en TIC deberían ser más frecuentes en las aulas, basado en la evolución desde los datos recogidos en estudios anteriores (eEurope 2005, eLearning 2006 y Eurydice), hecho que nos indica que la integración de las TIC está detenida en el tiempo o estancada.

\section{Método}

A partir de los resultados de The Survey of Schools surgió la motivación de realizar un estudio en dos etapas, con la aplicación de la Encuesta Escolar: Educación y TIC en la Región de Murcia (EEETRM) en la primera etapa, para definir la situación de la integración de la tecnología en los centros escolares de Educación Primaria de la Región de Murcia. En la segunda etapa, que no abarcamos en este artículo, se presentan los principales hallazgos de la encuesta a un grupo de discusión constituido por directores y docentes de la población encuestada para extraer las debilidades, amenazas, fortalezas y oportunidades de la integración de la tecnología en los centros de Educación Primaria de la Región de Murcia (Céspedes y Ballesta, 2018a; 2018b). Durante toda la investigación se tuvieron presentes los principios básicos de la investigación ética como el consentimiento informado; el compromiso con la honestidad y el cuidado de los participantes al reportar los hallazgos; y la gestión de los sesgos y posicionamiento del investigador (Wood y Smith, 2018, p.24).

\section{Muestra}

La encuesta se dirige a tutores de $4^{\circ}$ curso y directores de centros públicos, concertados y privados de Educación Primaria de la Región de Murcia. Teniendo en cuenta que la aplicación de la encuesta es online y contando con una población de 490 centros se optó por no realizar ningún tipo de muestreo e incluir al total de la población. De los 490 centros encuestados se obtuvo respuesta de 249 directores, una participación del 51\% que, en caso de haber realizado muestreo aleatorio simple supondría un nivel de confianza del $97 \%$ y un margen de error del 4,83\% asegurando las condiciones de generalización de datos (confia- bilidad, variabilidad y error). En cuanto a los tutores de $4^{\circ}$ de Educación Primaria, según los datos del Servicio de Estadística de la Consejería de Educación de la Región de Murcia, según la cantidad de centros y la media de líneas; y teniendo presente las unidades mixtas existentes en la Región, la población sería de aproximadamente 800 en el momento en el que se aplica la encuesta. Se obtuvo respuesta de 245 tutores, una participación del $30 \%$ que, haciendo la misma similitud que se ha hecho con la respuesta de directores, supondría un nivel de confianza del $97 \%$ con un margen de error del 5,8\% asegurando igualmente las condiciones de generalización de datos.

\section{Instrumentos}

En este estudio se han aplicado los cuestionarios de The Survey of Schools construidos, validados y pilotados en escuelas de Francia y Gran Bretaña antes de ser traducidos a 23 idiomas y publicados online para la aplicación de la encuesta. En nuestro estudio se aplicaron los cuestionarios de directores y tutores para el nivel ISCED 1, equivalente a $4^{\circ}$ de Educación Primaria.

Las dimensiones desarrolladas en el cuestionario se fundamentan en informes internacionales (Scheuermann y Pedró, 2009) teniendo en cuenta las prioridades formuladas por la Comisión Europea respecto a la Agenda Digital Europea i2020 (European Commission, 2014). Estas áreas, donde se ubican las variables del estudio, son las siguientes:

- Infraestructura de las TIC en las escuelas.

- Acceso a las TIC en la escuela por parte de estudiantes y profesores, así como las actividades basadas en las TIC.

- Confianza declarada de profesores sobre sus competencias digitales.

- Estrategia de las escuelas y liderazgo.

- Opiniones y actitudes de directores y profesores sobre las TIC.

El cuestionario de directores consta de 28 ítems repartidos en diez bloques (tabla 1) mientras que el de docentes cuenta con 31 ítems distribuidos en once bloques (tabla 2).

Dado que estos cuestionarios se aplicaron en España en The Survey of Schools se dio por validado el contenido y los procedimientos de escala de los ítems utilizados para tal fin, por tanto, no se efectuaron modificaciones para poder así realizar un análisis comparativo fiable a partir de los datos obtenidos por el estudio europeo.

Tabla 1. Variables analizadas en el cuestionario de directores. Fuente: elaboración propia

\begin{tabular}{lc}
\multicolumn{1}{c}{ Bloque } & Ítems \\
Identificación & 3 \\
Población escolar & 5 \\
Ubicación del centro & 1 \\
Infraestructura del centro & 8 \\
Apoyo a los docentes que utilizan las TIC & 2 \\
Obstáculos para el uso de las TIC en la enseñanza y el aprendizaje & 1 \\
Estrategia del centro para el uso pedagógico de las TIC & 3 \\
Opiniones sobre el uso pedagógico de las TIC & 1 \\
Uso del ordenador por parte del director o directora del centro & 1 \\
Información personal & 3
\end{tabular}


Tabla 2. Variables analizadas en el cuestionario de docentes. Fuente: elaboración propia

\begin{tabular}{lc}
\multicolumn{1}{c}{$\quad$ Bloque } & Ítems \\
Identificación & 2 \\
Información sobre el grupo al que imparte clase & 4 \\
Experiencia docente con las TIC & 3 \\
Acceso a las TIC para dar clase & 5 \\
Apoyo a los docentes que utilizan las TIC & 5 \\
Actividades basadas en las TIC y material para la enseñanza & 2 \\
Obstáculos para el uso de las TIC en la enseñanza y el aprendizaje & 1 \\
Actividades de aprendizaje con la clase & 1 \\
Habilidades docentes & 1 \\
Opiniones y actitud de los docentes & 2 \\
Información personal & 5
\end{tabular}

\section{Procedimiento}

La encuesta se aplicó entre mayo y julio de 2015 íntegramente online, a través del Servicio de Encuestas de la Universidad de Murcia con colaboración de la Consejería de Educación de la Región de Murcia, a través de la Dirección General de Calidad Educativa, Innovación y Atención a la Diversidad del Servicio de Evaluación y Calidad Educativa y de la Dirección General de Planificación Educativa y Recursos Humanos además de la Asociación de Directivos de Colegios Públicos en la Región de Murcia.

El tratamiento y análisis de datos se llevó a cabo por medio del software estadístico IBM SPSS Statistics, recorriendo las fases de análisis descriptivo, comparativo y correlacional. Para el estudio correlacional se ha utilizado el coeficiente de Spearman, al ser el más conveniente por la naturaleza de las variables. En cuanto a la significación estadística, como suele ser habitual en Ciencias
Sociales, hemos utilizado como niveles de significación .001 y .005 (Vilá y Bisquerra, 2014; Colás y Buendía, 1998).

\section{Resultados}

Provisión de infraestructura

La ratio de alumnos por ordenador y portátil en la Región de Murcia es de 8 alumnos por ordenador de sobremesa y 42 alumnos por dispositivo portátil, considerándose estos dispositivos no solo los ordenadores portátiles sino también netbooks, notebooks y tablets. Este dato nos indica que la Región de Murcia está por debajo de la media española, pero cerca de la media europea en cuanto a ordenadores de sobremesa y al referirnos a dispositivos portátiles esta media se sitúa muy por debajo de la nacional y de la europea (Figura 1).

Figura 1. Alumnos por portátiles y equipos de sobremesa.

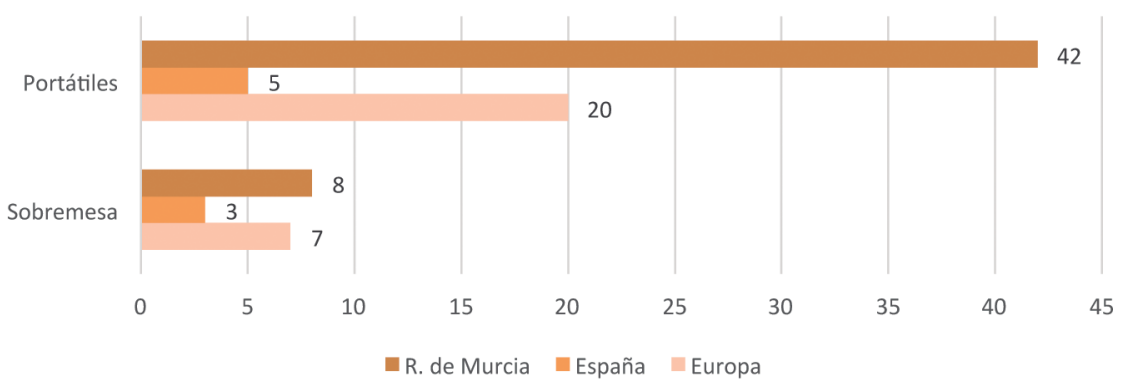

En la Región de Murcia lo habitual es encontrar un ordenador por aula, para el uso del profesor, junto a la pizarra digital interactiva; y varios ordenadores, dependiendo del número de líneas del centro, en una o varias aulas de informática.

El mantenimiento de los equipos depende del Responsable de Medios Informáticos o Coordinador TIC, que es el encargado de esta función en un $85 \%$ de los casos. En cuanto a la conectividad de los centros, entendida esta como todos los elementos que hacen posible la inter e intraconexión de los centros, es que, tal como se observa en la figura 2, pocos centros de Educación Primaria de la Región de Murcia (29\%) disponen de entornos virtuales de aprendizaje y menos aún (16\%) utilizan el correo electrónico con sus alumnos. Sin embargo, sí que está extendido el uso del email del profesorado (86\%) y la existencia de una web de centro (86\%).

En el análisis correlacional dentro de las variables de esta dimensión no se encuentra relación significativa entre el porcentaje de estudiantes de medios desfavorecidos y el número de ordenadores con acceso a internet ( $\mathrm{r}=-.005 ; \mathrm{p}>.05)$, y tampoco entre la provisión de pizarras digitales interactivas y el porcentaje de estudiantes de medios desfavorecidos ( $r=-.075 ; \mathrm{p}>.05)$. 
Figura 2. Conectividad en los centros de la Región de Murcia.

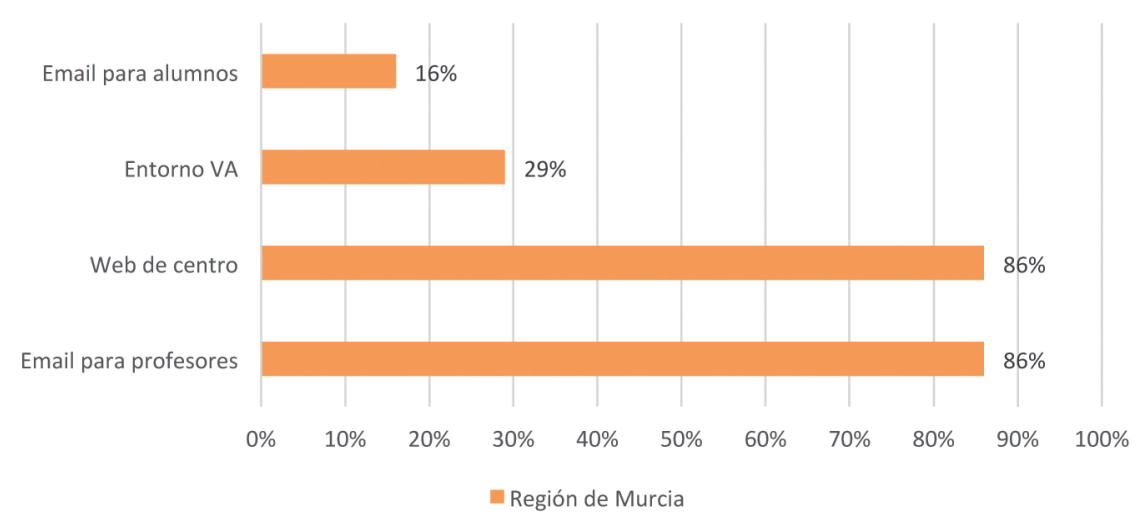

Uso de la infraestructura

Los obstáculos que encuentran los docentes para el uso de la tecnología en actividades de enseñanza y aprendizaje se agrupan en tres categorías:

- Los referentes al equipamiento.

- Los denominados pedagógicos (falta de competencia digital docente, insuficiente suporte técnico y pedagógico, falta de contenidos apropiados para integrar la tecnología a nivel de currículo o a nivel de aula o la falta de modelos para usar la tecnología en la enseñanza).

- Los denominados obstáculos de objetivo o meta (objetivos que padres, docentes y directores tienen respecto a la educación de sus hijos).

Directores y profesores coinciden en apuntar a los factores relacionados con el equipamiento como los más determinantes a la hora de obstaculizar el uso de la tecnología en las aulas.

No se encuentra relación significativa entre el nivel económico de las familias y la percepción de obstáculos pedagógicos por parte de los directores ( $\mathrm{r}=.030 ; \mathrm{p}>.05)$, pero sí que existe relación significativa positiva entre la percepción de los obstáculos rela- cionados con los objetivos $(\mathrm{r}=.126 ; \mathrm{p}<.05)$ y curiosamente al contrario que en The Survey of Schools, significación negativa con respecto a los obstáculos relacionados con el equipamiento (r=-.127; $\mathrm{p}<.05)$. Teniendo en cuenta las orientaciones de Bisquerra (2014), la correlación es prácticamente nula, y atendiendo al coeficiente de determinación, el $2 \%$ de los casos de alumnos que provienen de entornos desfavorecidos explica la percepción de obstáculos relacionados con aspectos pedagógicos o de objetivos por parte de los directores.

\section{Actividades basadas en TIC}

Los profesores usan principalmente la tecnología para preparar tareas de clase, navegar en Internet para preparar las sesiones y buscar material para usarlo en las sesiones. Las actividades basadas en TIC que se dan con menos frecuencia son la comunicación online con padres; evaluar y proporcionar retroalimentación a estudiantes; y poner tareas online. Como podemos observar en la figura 3, la frecuencia de actividades basadas en TIC es similar a la media europea y nacional situándose en varias veces al mes (valor 2 en la escala).

Figura 3. Frecuencia de actividades basadas en TIC.

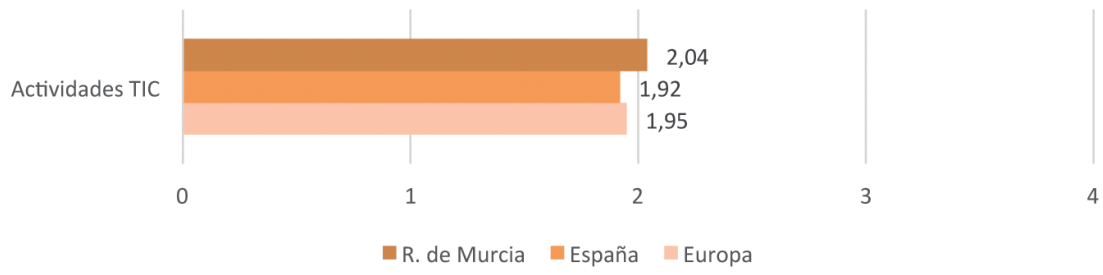

Para poder establecer correlaciones respecto a las actividades basadas en tecnología en las aulas se preguntó a los profesores sobre los estilos de enseñanza, diferenciando entre enseñanza centrada en el estudiante y enseñanza centrada en el profesor.

En el análisis correlacional de las variables de esta dimensión encontramos una relación significativa y positiva entre la frecuencia de actividades basadas en TIC en el aula y los estilos de aprendizaje centrados en el alumno ( $\mathrm{r}=.332 ; \mathrm{p}<.01)$. Esto significa que en centros donde se llevan a cabo metodologías centradas en el alumno la frecuencia de actividades basadas en TIC es mayor. Según el coeficiente de determinación, el 11\% del uso frecuente de TIC en el aula estaría asociado a los estilos de enseñanza cen- trados en el alumno por lo que podemos hablar de una correlación significativa, pero no muy alta.

\section{Desarrollo profesional y confianza en el uso de las TIC}

En España y Europa un 30\% de los docentes afirma que ha recibido formación en tecnología educativa durante su formación inicial. En la Región de Murcia este porcentaje es de un 18\%. Respecto a las vías de desarrollo profesional, destaca principalmente la formación en el tiempo libre, seguida de la formación en el mismo centro de trabajo y la participación en comunidades de aprendizaje online (figura 4). 
Figura 4. Vías de desarrollo de la competencia digital.

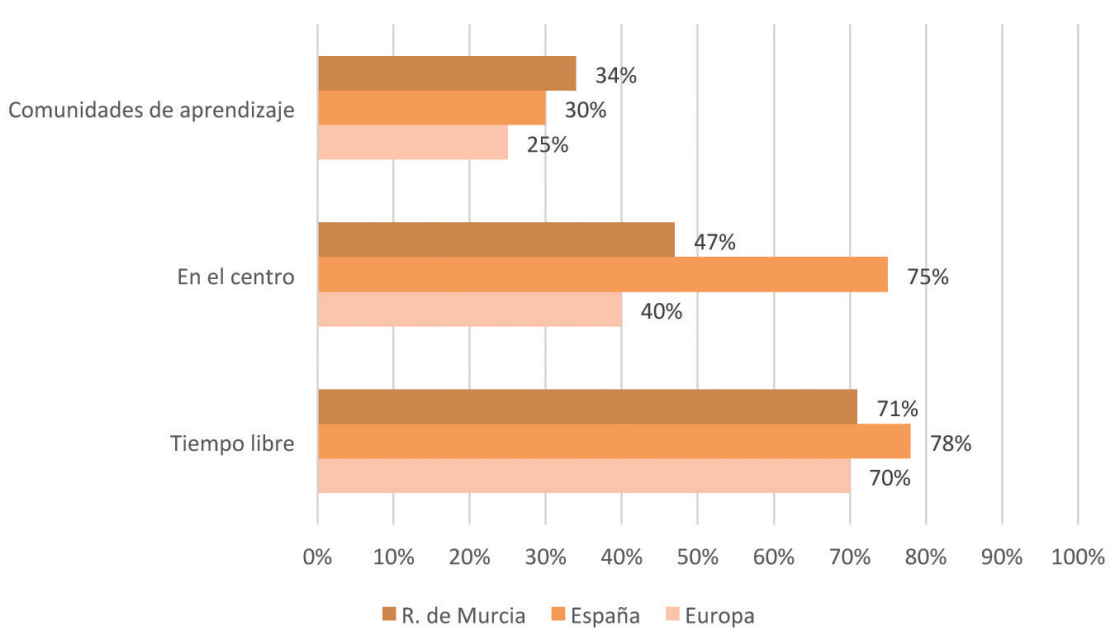

Los docentes se forman en el uso de equipamiento específico; cursos de introducción a internet y aplicaciones generales; y

cursos sobre el uso pedagógico de las TIC. Los cursos avanzados son los que menos porcentaje arrojan (figura 5).

Figura 5. Campos de desarrollo profesional relacionado con las TIC.

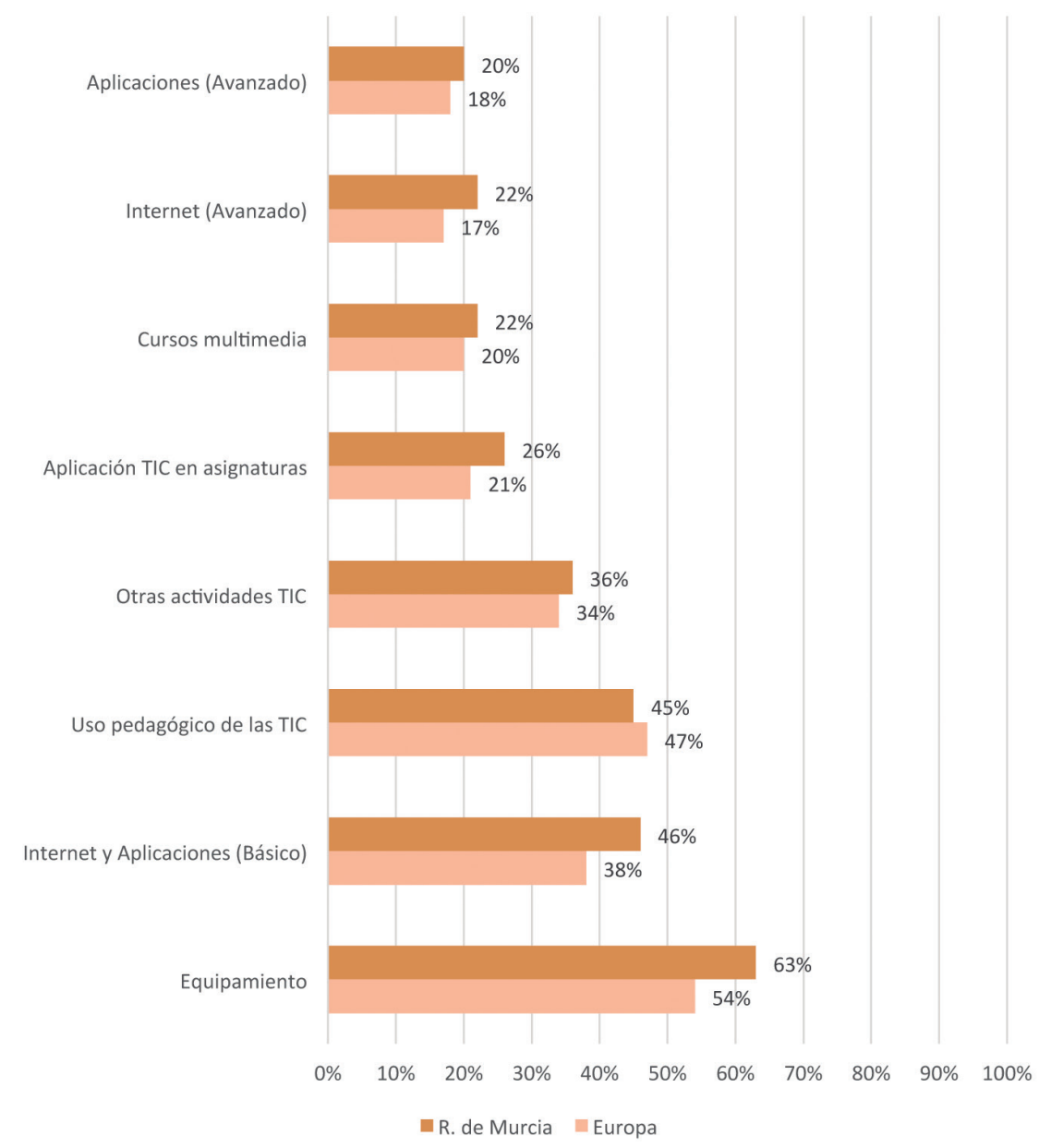

Aula Abierta, volumen 47, nº 3, julio-septiembre, 2018, págs. 355-364 
Los docentes tienen más confianza en sus habilidades operacionales, aquellas que tienen que ver con el uso de procesadores de textos, hojas de cálculo, sistemas operativos, etc., que, en sus habilidades mediáticas, las que tienen que ver con el uso de internet, blogs, etc.

Encontramos relación significativa entre la confianza de los profesores en habilidades operacionales y la participación en actividades de desarrollo profesional $(r=.390 ; \mathrm{p}<.01)$. Esto quiere decir que el hecho de que los profesores participen en actividades de desarrollo profesional relacionadas con las TIC aumenta levemente su confianza en estas habilidades.
Políticas, estrategias, incentivos y soporte de los centros

El porcentaje de centros en la Región de Murcia que programa reuniones en las que los docentes intercambian, evalúan y crean materiales didácticos, así como distintos enfoques para integrar las TIC en el aula (39\%) difiere respecto a la media de Europa (53\%). En cuanto a las estrategias específicas de cooperación y colaboración entre docentes, las medias de la Región y de Europa son similares (Figura 6). Respecto a los incentivos por el uso de las TIC con fines educativos, en la Región de Murcia no se percibe dicha incentivación (figura 7).

Figura 6. Estrategias específicas en materia de TIC en los centros.

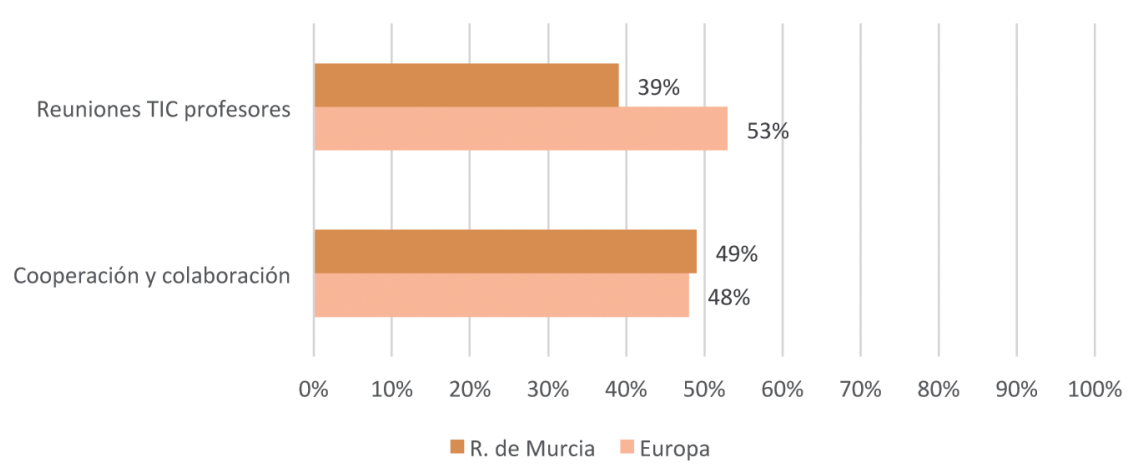

La figura del Coordinador TIC en la Región de Murcia, bajo la denominación de Responsable de Medios Informáticos, es la representación del soporte tecnológico a nivel de centro y aula con presencia en todos los centros de Educación Primaria de la Re- gión de Murcia y de España, sin embargo, en Europa la figura del Coordinador TIC está presente en un $62 \%$ de los centros educativos de este nivel. Tal como refleja la figura 8, el Responsable de Medios Informáticos no siempre presta apoyo pedagógico (52\%).

Figura 7. Incentivos por el uso de las TIC en las aulas.

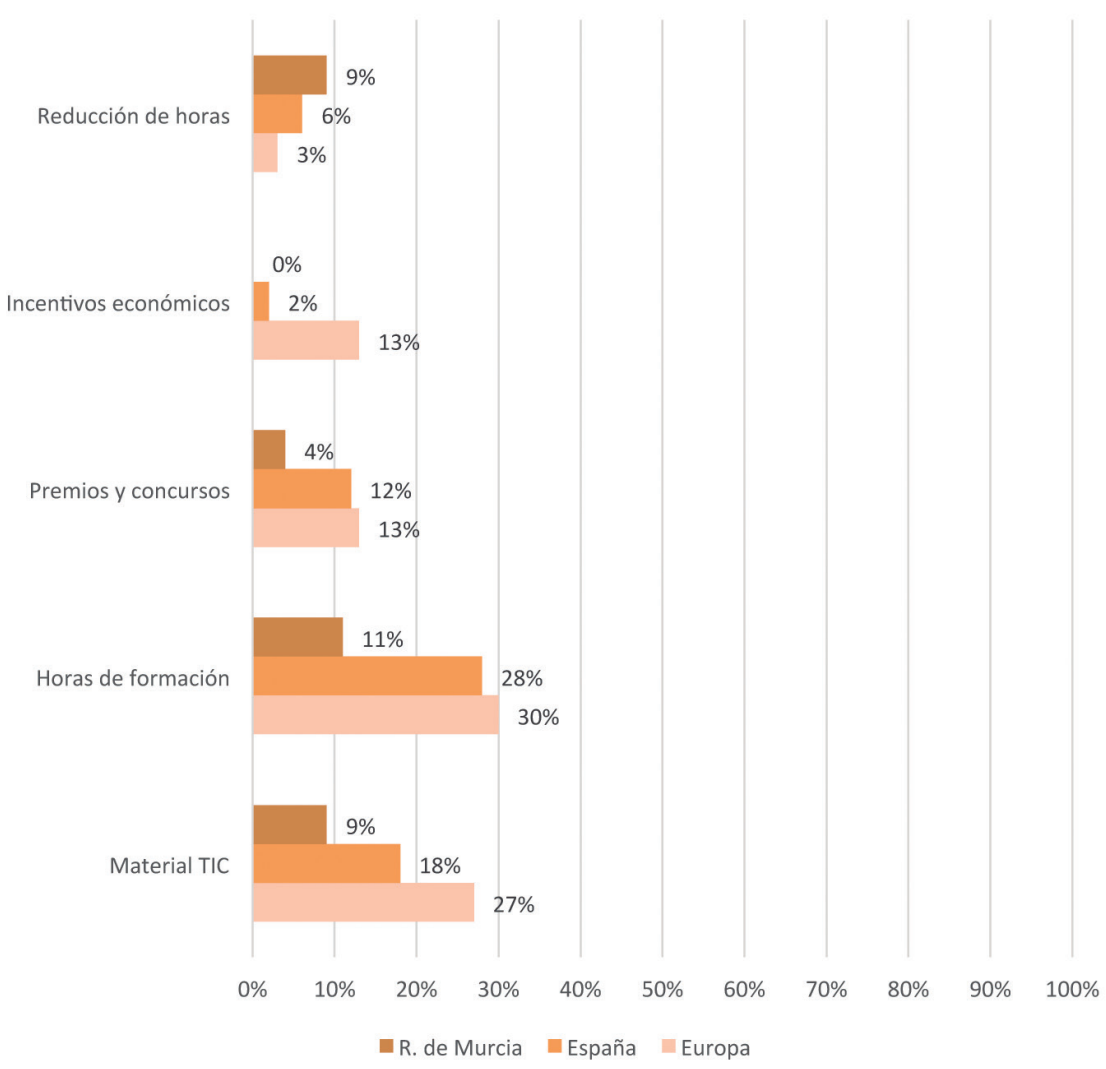


Figura 8. Figura del Responsable de Medios Informáticos.

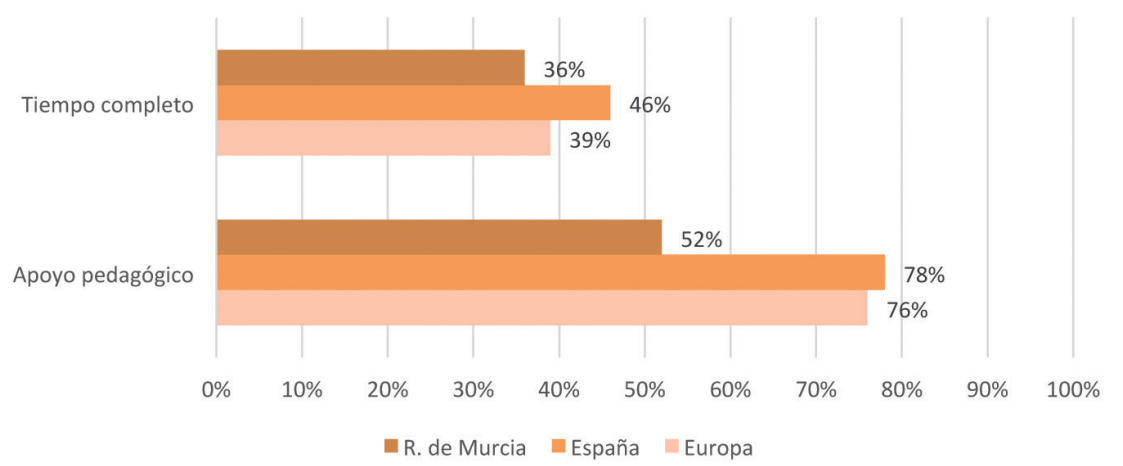

Actitudes y opiniones sobre el uso pedagógico de las TIC

La tecnología es percibida como un elemento motivador en las aulas y se utiliza fundamentalmente para recoger información de Internet. Tanto directores como docentes están de acuerdo en que el uso de tecnología en las aulas favorece el trabajo colaborativo y tiene un efecto positivo en el trabajo autónomo. Se observa un amplio acuerdo en que la tecnología tiene efectos positivos en los procesos de enseñanza y aprendizaje, con porcentajes por encima del $90 \%$ en ambos colectivos. Asimismo, hay consenso al afirmar que la tecnología es necesaria para formar a los alumnos del Siglo XXI y que es necesario cambiar radicalmente la educación para poder explotar al máximo el potencial de la tecnología en los centros educativos. Al preguntar a los docentes sobre la visión compartida con compañeros, dirección y demás personal del centro sobre el enfoque de la integración de las TIC en el aprendizaje, se obtiene una respuesta positiva en el $43 \%$ de los docentes.

En el análisis correlacional no encontramos relación significativa entre la actitud de los directores en cuanto al uso de las TIC en la enseñanza y el aprendizaje y su género, edad, años de experiencia y tipo de zona del centro. Sí se encuentra correlación positiva significativa con el porcentaje de alumnos de medios desfavorecidos en el centro $(r=.170 \mathrm{p}<.01)$ que nos indica que, a más porcentaje de alumnos en desventaja económica en el centro, peor es la actitud de los directores respecto al uso de las TIC en el aula. Esta correlación es prácticamente nula al encontrarse entre 0 y .20 según Bisquerra (2014), y cuenta con un coeficiente de determinación del 3\%. Se encuentra correlación negativa significativa respecto al tiempo dedicado al perfeccionamiento profesional $(\mathrm{r}=-.129 \mathrm{p}<.05)$ con un coeficiente de determinación del $2 \%$ que nos indica que esta variable está débilmente relacionada con la opinión desfavorable sobre el uso pedagógico de las TIC. Se encuentra correlación positiva respecto al porcentaje de uso de las TIC en clase $(r=.196 \mathrm{p}<.01)$ con un coeficiente de determinación del $4 \%$ que nos indica que muy débilmente a mejor opinión sobre las TIC más tiempo se usa la tecnología en el aula. Respecto a la confianza en sus habilidades operacionales ( $\mathrm{r}=.156$ $\mathrm{p}<.05)$ y mediáticas $(\mathrm{r}=.174 \mathrm{p}<.01)$, encontramos correlaciones positivas significativas, con coeficientes de determinación entre el $2 \%$ y $3 \%$ que indica que, también débilmente, cuanto mejor es su opinión, más confiados se sienten con las TIC. Respecto al enfoque compartido sobre el uso de las TIC en los centros ( $\mathrm{r}=.156$ $\mathrm{p}<.05$ ) encontramos correlación positiva y significativa, con un coeficiente de determinación que indica que el 2\% de los casos de centros que comparten su visión sobre el uso de las TIC está explicado por la opinión favorable de los docentes en cuanto al uso de las TIC en la enseñanza y el aprendizaje.

\section{Discusión y conclusiones}

Los centros de Educación Primaria de la Región de Murcia están suficientemente dotados de pizarras digitales interactivas y ordenadores de sobremesa, pero escasean los dispositivos portátiles que de forma generalizada se recomiendan en el ámbito educativo (Haßler, Major, y Hennessy, 2016; Biagi y Loi, 2013; Norris, Hossain y Soloway, 2011; Redecker y Johannessen, 2013). Cada vez se enfatiza más en la necesidad de incrementar la provisión de portátiles y tablets en las escuelas para mejorar tanto los procesos de aprendizaje como las competencias de los estudiantes y existen evidencias acerca de la relación entre los dispositivos portátiles y la motivación, el desarrollo de habilidades de organización, aumento de la responsabilidad, fomento del aprendizaje autónomo y colaborativo, así como su utilidad para la evaluación del progreso de los alumnos (Savill-Smith, 2005; Alderete, Di Meglio y Formichella, 2017; Bocconi et al., 2013; Camacho y Esteve, 2018; Redecker y Johannessen, 2013). La dotación de pizarras digitales interactivas es un dato positivo, pero algunos estudios (Underwood, 2009) cuestionan el papel transformador en la educación ya que no llegan a conectar con pedagogías potencialmente transformadoras (Wastiau, 2010). Cabe destacar en este punto que, tanto en The Survey of Schools como en la Encuesta Escolar, se observa que un gran número de equipos se encuentran ubicados en las aulas de informática comprometiendo así el acceso a los mismos. Corroboramos el escaso uso de entornos virtuales de aprendizaje que aportan también un grado importante de ubicuidad de la enseñanza y el aprendizaje (Wastiau et al. 2013; Vázquez-Cano y Sevillano, 2015). Aún se cuenta con una conectividad pobre en la que no se hace uso del correo electrónico de alumnos ni de entornos virtuales de aprendizaje en la escuela, a pesar de disponer de ambos servicios a través de la Consejería de Educación desde hace más de una década, y teniendo en cuenta que la formación online actualmente supone una vía de desarrollo profesional fomentada y utilizada en múltiples modalidades.

Son muy pocos los profesores que usan la tecnología a la vez con los alumnos en clase, y menos para comunicarse con la familia o repartir el trabajo entre la escuela y el hogar a través de metodologías como la Flipped Classroom (Wastiau et al., 2013). En estudios como el de Alderete et al. (2017) se afirma que el acceso a la tecnología en el hogar mejora el rendimiento escolar, pero esas bondades no se reproducen en el interior de la escuela, hecho que podría explicarse por la brecha de acceso de algunos estudiantes por falta de recursos o por un uso indebido de la tecnología en los centros como el concentrarla en aulas de informática que hay que reservar previamente para acceder o que ya tienen un horario restringido y establecido desde el comienzo 
de curso. Portátiles, smartphones y lectores digitales no existen o carecen de accesibilidad, y tardarán aún en implementarse en las aulas (Pérez Tornero y Pi, 2014) a pesar de que, en el caso de los smartphones son el medio más utilizado para acceder a internet actualmente (Fundación Telefónica, 2017).

Las metodologías centradas en el alumno y la frecuencia de uso de actividades basadas en TIC están relacionadas, por lo que podemos afirmar que las TIC son herramientas con potencial transformador, aunque ese potencial esté aún por explotar dado que la extensión de la enseñanza tradicional es aún predominante.

Directores y profesores apuntan al número insuficiente de ordenadores y al estado de los mismos, obsoletos o estropeados, como los mayores obstáculos que encuentran a la hora de realizar actividades basadas en TIC en el aula. Al igual que destacan la falta de apoyo, tanto técnico como pedagógico. Ambos colectivos apuntan a los obstáculos relacionados con el equipamiento como más determinantes que los pedagógicos o didácticos, seguramente debido a que los primeros dificultan explorar el campo del uso pedagógico de la tecnología (Pelgrum, 2001).

La integración eficaz de la tecnología en las aulas depende del desarrollo profesional y la confianza de los docentes en sus habilidades con las TIC (Fredriksson, Jedeskog \& Plomb., 2008; Valiente, 2011; Wastiau et al., 2013). Aunque las universidades españolas cuentan con una escasa y desigual formación inicial en tecnología educativa en sus planes de estudio (Ballesta y Céspedes, 2015), los docentes no perciben estar suficientemente preparados en su formación inicial (OCDE, 2008; Starkey, 2010), lo que indica que debe hacerse una revisión de los planes de estudio en las universidades respecto al uso pedagógico de las TIC. La mayor parte de los docentes se desarrolla profesionalmente en su tiempo libre a través de métodos informales de formación, formación semipresencial y formación relacionada con situaciones reales de aula, cercanas a la realidad y con aplicación práctica (Balanskat, Blamire \& Kefala, 2006). La formación realizada junto al personal del centro de trabajo aún no está muy extendida a pesar de que estos escenarios son apoyados en muchos estudios (Escudero, 2011; Escudero, González y Rodríguez, 2013). Se correlaciona positivamente el desarrollo profesional con la confianza de los docentes en el uso de las TIC y el aumento de confianza con un aumento en la frecuencia de actividades basadas en TIC que a su vez podría favorecer estilos de enseñanza centrados en el alumno. Todas estas relaciones son débiles, pero resultaría interesante estudiar vías para fortalecerlas si queremos incrementar el protagonismo de los alumnos en las escuelas.

Las políticas educativas, a cualquier nivel, deben fomentar el trabajo colaborativo entre profesores e incentivar el uso de repositorios o bases de datos bien organizadas y categorizadas, que podrían ser altamente beneficiosas para el profesorado (Bacigalupo y Cachia, 2011). El fomento de conversaciones periódicas entre docentes sobre el uso pedagógico de la tecnología, hemos comprobado que está bastante extendida en Europa y que junto a la cooperación entre docentes supone un factor clave para su desarrollo profesional y sus implicaciones en la integración de las TIC en la enseñanza (Bacigalupo y Cachia, 2011; Escudero, 2011, 2012). Por otra parte, el uso de las TIC en los centros no parece estar suficientemente incentivado, mientras que en Europa se ofrece material TIC adicional para clase y horas de formación, incentivos que se dan también a nivel nacional y que, sin embargo, en la Región de Murcia no son percibidos.

El asesoramiento o soporte que reciben los docentes para el uso de las TIC es un factor clave para asegurar la correcta integración de las mismas. De los datos de la Encuesta Escolar se extrae que uno de cada cuatro docentes afirma no recibir ni apoyo pedagógico, ni técnico del Responsable de Medios Informáticos el cual ocupa gran parte de su tiempo en el mantenimiento de los equipos sin ser técnico profesional, función que se asignó en un principio a este cargo pero que debía haber evolucionado tras la entrada en escena de la escuela 2.0 pasando de una focalización en el hardware a un papel dinamizador de las TIC (Garrido, Fernández y Sosa, 2008; Espuny, Gisbert, Coiduras y González, 2012). Teniendo en cuenta todo lo expuesto y que, atendiendo a los datos de The Survey of Schools, la figura del Coordinador TIC no existe en un considerable número de centros de Educación Primaria de Europa nos cuestionamos la continuidad de este puesto que, tras casi dos décadas en nuestros centros, necesitaría una revisión según las necesidades, estrategias y políticas actuales.

Entre otras ventajas, directores y profesores afirman que la tecnología motiva y es muy útil para buscar información. Sin embargo, no todos están de acuerdo en afirmar que mejora el rendimiento de los alumnos o las habilidades de orden superior, por lo que podemos afirmar que la actitud en los centros es crítica, pero muy positiva, por lo que no suponen un obstáculo para la integración de la tecnología en las aulas. Esta actitud favorable es necesaria para que el potencial de las TIC lleve a mejoras en el aprendizaje de los alumnos (Abachi y Muhammad, 2014; Del Moral, Villalustre y Neira, 2014), ya que el buen uso de las TIC podría estar relacionado con la percepción de utilidad y confianza en el propio uso de la tecnología por parte del profesorado (Siddiq, Scherer y Tondeur, 2016).

A partir de los resultados obtenidos en la Encuesta Escolar: Educación y TIC en la Región de Murcia, comparados con los resultados de The Survey of Schools en Europa, podemos enumerar una serie de recomendaciones tanto a nivel de administración educativa como a nivel de liderazgo en centros educativos dentro de su alcance en el establecimiento de estrategias y políticas para la integración de la tecnología en los procesos de enseñanza y aprendizaje:

$\S$ Dispositivos portátiles en los centros: Sería necesario centrarse en políticas a nivel local sobre el uso de las TIC en los centros para reducir la brecha entre el uso de tecnología en la escuela y el uso que se le da en los hogares. En esta línea, observamos que los alumnos apenas usan ordenadores de sobremesa fuera de la escuela, son usuarios de dispositivos móviles. La eliminación de Aulas de Informática como espacios de concentración de tecnología y la sustitución de ordenadores de sobremesa por dispositivos portátiles podría ser doblemente útil, al ganar espacios para los centros y ser más coherentes con el uso de la tecnología por parte de los alumnos fuera de la escuela. Otra opción interesante sería estudiar la viabilidad de experiencias como la de que los alumnos lleven al centro sus propios dispositivos. No podemos olvidar las diferentes condiciones de educabilidad de los estudiantes. Sería recomendable establecer estrategias que compensen la falta de recursos de algunos estudiantes a modo de becas, préstamos o entrega de equipos informáticos a los alumnos más necesitados.

$\S$ Incentivos por el uso eficaz de las TIC en las aulas: Incentivar de forma efectiva el uso de las TIC en el aula en forma de premios o dotando a los centros de material adicional podría motivar a los docentes a usar la tecnología con fines pedagógicos, pero pensamos que ese reconocimiento debe ser meticuloso, premiando experiencias verdaderamente eficientes y efectivas que aporten resultados claros y no se queden en experiencias efímeras y puntuales. La incorporación de tecnología en las aulas debe perseguir un objetivo concreto y cumplirlo con éxito sin generar más trabajo ni ocupar más tiempo del que dispone el profesorado. 
$\S$ Fomento de entornos virtuales de aprendizaje: Se debe informar y formar al profesorado en el uso de entornos virtuales de aprendizaje y modalidades de enseñanza alternativas y complementarias a la presencial ya que suponen una vía de formación y desarrollo profesional que se usa cada vez más entre profesores y estudiantes. Este tipo de plataformas puede contribuir en las medidas de atención a la diversidad, por ejemplo, como soporte para contenidos de ampliación o refuerzo, pero también se ha demostrado su eficacia al traspasar la escuela los límites del edificio donde se imparten las clases ordinarias mejorando la relación entre alumnos, profesor y familias.

$\S$ Revisión de la formación inicial docente: Sabemos que este punto es bastante complejo, pero los datos revelan que la formación en tecnología educativa de nuestros docentes en la Universidad debe mejorar bastante, dotando de más importancia a la enseñanza de la tecnología educativa. En caso de optar por asignaturas específicas, deben ser impartidas por docentes que estén vinculados a una práctica real en las aulas, y un conocimiento profundo y actualizado de la materia. En el caso de ofrecer la tecnología educativa como materia transversal sería necesario contar con asesoramiento de docentes que ya integren con éxito la tecnología en sus centros. En otras palabras, hace falta conectar directa y realmente la Universidad con la realidad en los centros educativos en materia de tecnología educativa.

§ Revisión de la figura del Responsable de Medios Informáticos: Los docentes necesitan apoyo en el uso pedagógico de las TIC en los centros. Este apoyo debe ser una realidad y los docentes deben percibirlo así, por lo que recomendamos revisar la figura del Responsable de Medios Informáticos o Coordinador TIC en busca de funciones que se perciban más efectivas y se alejen de las labores exclusivamente técnicas.

$\S$ Continuidad en el uso de tecnología en los centros: Los problemas técnicos con la infraestructura en los centros son una realidad y un lastre importante al intentar programar actividades basadas en tecnología en las aulas. Es necesario aportar soluciones ágiles y rápidas que permitan usar a los docentes los equipos de forma continua, sin interrupciones ni contratiempos como se dan a diario en los centros. La solución a este problema pensamos que puede ser una opción mixta en la que los profesores asuman el mantenimiento de equipos propios que lleven a las aulas o sean subvencionados para tal fin; y un servicio técnico que se haga cargo del mantenimiento general o asesoramiento telemático a docentes para la solución ágil de los problemas cotidianos.

$\S$ Observatorio de innovación educativa: Sería interesante crear un observatorio oficial de prácticas educativas, con o sin tecnología, que periódicamente hiciese un seguimiento de prácticas que fuesen efectivas, con base científica, en términos de eficiencia y eficacia. Por otra parte, este observatorio acercaría la estadística al colectivo docente, alejándoles de la crítica sin fundamento y dándole el valor objetivo que tiene. Este observatorio contribuiría al desarrollo en el profesorado de una actitud crítica y fundamentada, y serviría para apuntar a necesidades reales poniendo el foco en las prácticas que, verdaderamente, pueden ser útiles, así como orientar, tanto a la administración educativa como a los centros, en el establecimiento de planes y acciones de mejora en los procesos de ensenanza y aprendizaje en las aulas.
A tenor de todo lo expuesto y a modo de síntesis, recomendamos marcar como objetivo prioritario que tanto profesores como alumnos perciban el uso de la tecnología en los centros igual que fuera de ellos sin que exista discontinuidad y logrando así que la tecnología sea una herramienta útil que sume en los procesos de enseñanza y aprendizaje. Evidentemente, nos sumamos a estudios como el de Camacho y Esteve (2018) en la recomendación de que la incorporación de tecnología debe ir acompañada de estilos de enseñanza centrados en el alumno, activos, y de estrategias didácticas que fomenten la colaboración y el trabajo en equipo que como se desprende de los resultados de la Encuesta Escolar son factores que de alguna manera están relacionados. Concluimos que, tal vez, la clave no sea llevar a cabo una gran inversión en equipos y mantenimiento, en tanto que podría ser más efectiva una buena coordinación y reparto de responsabilidades entre asesores técnicos, asesores pedagógicos, docentes y estudiantes con respecto inmersión tecnológica en las aulas a favor de un uso coherente de la tecnología como recurso eficaz y eficiente al mismo tiempo.

\section{Referencias bibliográficas}

Abachi, H. R., y Muhammad, G. (2014). The impact of m-learningtechnology on students and educators. Computers in human behavior, 30, 491-496.

Alderete, M. V., Di Meglio, G. y Formichella, M. M. (2017). Acceso a las TIC y rendimiento educativo: ¿una relación potenciada por su uso? Un análisis para España. Revista de Educación, $377,54-81$.

Area, M. (2010). El proceso de integración y uso pedagógico de las TIC en los centros educativos. Un estudio de casos. Revista de Educación, 352, 77-97.

Bacigalupo, M. y Cachia, R. (2011). Teacher Collaboration Networks in 2025. What is the Role of Teacher Networks for Professional Development in Europe?: Sevilla, JRCIPTS

Balanskat, A., Blamire, R. y Kefala, S. (2006). The ICT impact report: a review of studies of ICT impact on schools in Europe. Brussels: European Schoolnet, European Commission.

Ballesta, J. y Céspedes. R. (2015). Los contenidos de Tecnología Educativa en las titulaciones de grado de las universidades españolas. RELATEC. Revista Latinoamericana de Tecnología Educativa, 14(1), 133-143.

Biagi, F. y Loi M. (2013). Measuring ICT Use and Learning Outcomes: evidence from recent econometric studies. European Journal of Education, 48(1), 28-42.

Bisquerra, R. (2014). Metodología de la investigación educativa. Madrid: Editorial La Muralla.

Bocconi, S., Panagiotis, K., y Punie, Y. (2013). Framing ICT-enabled innovation for Learning: the caso of one. European Journal of Education, 48(1), 113-130.

Camacho, M. y Esteve, F. M. (2018). El uso de las tabletas y su impacto en el aprendizaje. Una investigación nacional en centros de Educación Primaria. Revista de Educación, 379, 170191.

Céspedes, R. y Ballesta, J. (2018a). Análisis de los factores externos a la integración de las TIC en Educación Primaria. En V. Abella, V. Ausín y V. Delgado (Eds.). XXV Jornadas Universitarias de Tecnología Educativa - JUTE 2017 (pp. 352-359). Burgos, España: Universidad de Burgos.

- (2018b). Análisis de los factores internos a la integración de las TIC en Educación Primaria. En D. Marín, Ma I. Pardo, I. Vidal y $\mathrm{M}^{\mathrm{a}} \mathrm{J}$. Waliño (Eds.). II Jornadas Tecnológicas de la Desregulación de Contenidos Curriculares (pp. 116-121). Valencia, España: Universidad de Valencia. 
Colás, P. y Buendía, L. (1998). Investigación Educativa. Sevilla: Ediciones Alfar.

Colás, P., De Pablos, J., y Ballesta, J. (2018). Incidencia de las TIC en la enseñanza en el sistema educativo español: una revisión de la investigación. RED. Revista de Educación a Distancia, 56. Recuperado de https://goo.gl/mhAvs7

Condie, R. y Munro, B. (2007). The impact of ICT in Schools- A Landscape Review. Coventry: BECTA. Recuperado de https:// goo.gl/hBN3Hv

De Pablos, J. (2010). Políticas educativas y la integración de las TIC a través de buenas prácticas docentes. En J. de Pablos, M. Area, J. Valverde y J. M. Correa (Coords.), Políticas educativas y buenas prácticas con TIC (pp. 21-41). Barcelona: Graó.

Del Moral, M. E., Villalustre, L. y Neira, M. R. (2014). Oportunidades de las TIC para la innovación educativa en las escuelas rurales de Asturias. Aula Abierta, 42(1), 61-67.

Escudero, J. M. (2012). La colaboración docente, una manera de aprender juntos sobre el trabajo cooperativo con el alumnado. En Torrego, J. C. y Negro, A. (Coords.). Aprendizaje cooperativo en las aulas. Fundamentos y recursos para su implementación. Madrid: Alianza Editorial.

Escudero, J. M. (2011). Los centros escolares como espacios de aprendizaje y de desarrollo profesional de los docentes. En González, M. T. (Coord.). Innovaciones en la Gestión y el Gobierno de los centros escolares. Madrid: Síntesis.

Escudero, J. M., González, M. T. y Rodríguez, M. J. (2013). La mejora equitativa de la educación y la formación del profesorado. Multidisciplinary Journal of Educational Research, 3(3), 206-234. Recuperado de https://goo.gl/TpBCxb

Espuny, C., Gisbert, M., Coiduras, J. y González, J. (2012). El coordinador TIC en los centros educativos: funciones para la dinamización e incorporación didáctica de las TIC en las actividades de aprendizaje. Pixel-Bit. Revista de Medios y Educación, 41, 7-18.

European Commission (2014). Digital agenda for Europe. Rebooting Europe's economy. Luxemburg: EU publications. DOI: $10.2775 / 41229$

- (2013). Survey of Schools: ICT in Education. Benchmarking Access, Use and Attitudes to Technology in Europe's Schools. DOI: $10.2759 / 944997$

Fundación Telefónica (2017). La Sociedad de la Información en España 2016. Madrid: Ariel.

Fredriksson, U., Jedeskog, G. \& Plomb, T. (2008). Innovative use of ICT in schools based on the findings in ELFE project, Education \& Information Technologies, 13, 83-101.d

Garrido, M. C., Fernández, R. y Sosa, J. M. (2008). Los coordinadores TIC en Extremadura. Análisis legislativo y valoración de su implantación en los centros educativos de primaria y secundaria en la region. Quaderns digitales. Eduteka. Recuperado de https://goo.gl/PaxNtM

Haßler, B., Major, L., y Hennessy, S. (2016). Tablet use in schools: a critical review of the evidence for learning outcomes. Journal of Computer Assisted Learning, 32(2), 139-156.

Norris, C., Hossain, A. y Soloway, E. (2011). Using smartphones as essential tools for learning. Educational Technology Magazine, 51(3), 18-25. Recuperado de https://goo.gl/lfgh8h

OCDE (2008). ICT and InitialTeacherTraining-research. Review Draft. Paris: OECD Publishing.
Pérez Tornero, J. M. y Pi, M. (Dir.) (2014). Perspectivas 2014. Tecnología y Pedagogía en las aulas. El futuro inmediato en España. Editorial Planeta. Recuperado de https://goo.gl/X7n4kG

Pelgrum, W. J. (2001). Obstacles to the integration of ICT in education: results from a worldwide educational assessment. Computers E Education, 37(2), 163-178.

Prensky, M. (2004). Digital Game-based Learning. New York: McGraw Hill.

Redeckter, Ch. y Johannessen, O. (2013). Changing Assessment-Towards a New Assessment Paradigm Using ICT. European Journal of Education, 48(1), 79-96.

Rodríguez, P., Nussbaum, M. y Dombrovskaia, L. (2013). ICT for education: a conceptual framework for the sustainable adoption of technology enhanced learning environments in schools. Technology, Pedagogy and Education, 21(3), 291-315.

Sancho, J. M. y Hernández, F. (2018). La profesión docente en la era del exceso de información y la falta de sentido. RED. Revista de Educación a Distancia, 56(4), 1-23. DOI: http://dx.doi. org/10.6018/red/56/4

Savill-Smith, C. (2005). The use of palmtop computers for learning: a review of the literature. British Journal of Educational Technology, 36(3), 567-568.

Scheuermann, F. y Pedró, F. (2009). Assessing the Effects of ICT in Education: Indicators, Criteria and Benchmarks for International Comparisons. Luxemburgo: OPOCE. DOI: 10.2788/27419

Siddiq, F., Scherer, R., y Tondeur, J. (2016). Teachers' emphasis on developing students' digital information and communication skills (TEDDICS): A new construct in 21st century education. Computers \& Education, 92, 1-14.

Schultz, J. (2017). How Much Data is Created on the Internet Each Day? Recuperado de https://goo.gl/S9ZuAk

Starkey, L. (2010). Teachers' pedagogical reasoning and action in the digital age. Teachers and Teaching: theory and practice, 16, 233-244.

Underwood, J. (2009). The impact of Digital Technology. Coventry: BECTA

Valiente, O. (2011). Los modelos 1:1 en educación. Prácticas internacionales, evidencia comparada e implicaciones políticas. Revista Iberoamericana de Educación, 56, 113-134.

Valle, J. y Manso, J. (2013). Competencias clave como tendencia de la política educativa supranacional de la Unión Europea. Revista de Educación, Número extraordinario 2013, 12-33.

Vázquez-Cano, E. y Sevillano, M. L. (2015). Dispositivos digitales móviles en educación. El aprendizaje ubicuo. Madrid: Narcea.

Vilà, R. y Bisquerra, R. (2014). El análisis cuantitativo de datos. En R. Bisquerra (coord.). Metodología de la investigación educativa. (pp. 260-271). Madrid: Editorial La Muralla

Wastiau, P. (2010). Virtual Learning Platforms in Europe: what can we learn from experience in Denmark, the United Kingdom and Spain? - A Comparative Overview. Paris/Bruselas: Caisse del Dépôts/European SchoolNet.

Wastiau, P., Blamire, R., Kearney, C., Quittre, V., Van de Gaer, E. \& Monseur, Ch. (2013). The Use of ICT in Education: a survey of schools in Europe. European Journal of Education, 48(1), 11-27.

Wood, P. y Smith, J. (2018). Investigar en educación. Conceptos básicos y metodología para desarrollar proyectos de investigación. Madrid: Narcea. 